\title{
Mastermind mediates chromatin-specific transcription and turnover of the Notch enhancer complex
}

\author{
Christy J. Fryer, ${ }^{1}$ Elise Lamar, ${ }^{2}$ Ivana Turbachova, ${ }^{1}$ Chris Kintner, ${ }^{2}$ and Katherine A. Jones ${ }^{1,3}$ \\ ${ }^{1}$ Regulatory Biology Laboratory and ${ }^{2}$ Molecular Neurobiology Laboratory, The Salk Institute for Biological Studies, \\ La Jolla, California 92037, USA
}

\begin{abstract}
Signaling through the Notch pathway activates the proteolytic release of the Notch intracellular domain (ICD), a dedicated transcriptional coactivator of CSL enhancer-binding proteins. Here we show that chromatin-dependent transactivation by the recombinant Notch ICD-CBF1 enhancer complex in vitro requires an additional coactivator, Mastermind (MAM). MAM provides two activation domains necessary for Notch signaling in mammalian cells and in Xenopus embryos. We show that the central MAM activation domain (TAD1) recruits $\mathrm{CBP} / \mathrm{p} 300$ to promote nucleosome acetylation at Notch enhancers and activate transcription in vitro. We also find that MAM expression induces phosphorylation and relocalization of endogenous $\mathrm{CBP} / \mathrm{p} 300$ proteins to nuclear foci in vivo. Moreover, we show that coexpression with MAM and CBF1 strongly enhances phosphorylation and proteolytic turnover of the Notch ICD in vivo. Enhanced phosphorylation of the ICD and p300 requires a glutamine-rich region of MAM (TAD2) that is essential for Notch transcription in vivo. Thus MAM may function as a timer to couple transcription activation with disassembly of the Notch enhancer complex on chromatin.
\end{abstract}

[Key Words: Notch; Mastermind; transcription activation; chromatin; phosphorylation; proteolysis]

Received March 15, 2002; revised version accepted April 22, 2002.

The Notch signaling pathway regulates important cell fate decisions, including neurogenesis and hematopoiesis (for reviews, see Greenwald 1998; Artavanis-Tsakonas et al. 1999; Mumm and Kopan 2000; Anderson et al. 2001). The transmembrane Notch receptors characteristically possess EGF- and Notch/Lin12-type repeats in the extracellular domain, as well as multiple ankyrin (ANK) repeats, a nuclear localization motif, and PEST control sequences in the intracellular domain. In the presence of cells expressing Delta or Jagged/Serrate ligands, the Notch receptor undergoes sequential proteolytic cleavage steps that ultimately release the intracellular domain (ICD) from the membrane. The untethered ICD enters the nucleus and targets members of the CSL (CBF1, Suppressor of Hairless, LAG-1) family of enhancer-binding proteins, providing an activation domain that enables the CSL proteins to activate transcription of Notch target genes. Identified targets of Notch signaling include basic helix-loop-helix proteins that antagonize cell differentiation, such as those encoded by the Drosophila Enhancer of split or mammalian Hairy enhancer of split genes.

${ }^{3}$ Corresponding author.

E-MAIL jones@salk.edu; FAX (858) 535-8194.

Article and publication are at http://www.genesdev.org/cgi/doi/10.1101/ gad.991602.
The Notch transactivation mechanism generally resembles that characterized for other inducible enhancers. Thus, Notch target genes are actively repressed in the absence of signaling by the association of CSL proteins with histone deacetylases and CtBP, SMRT, or CIR corepressors (Dou et al. 1994; Kao et al. 1998; Hsieh et al. 1999; Morel et al. 2001). CBF1 may also repress basal transcription through binding to TFIIA (Olave et al. 1998). Several other important components of the Notch enhancer complex have been identified genetically and biochemically, including Mastermind (MAM; Smoller et al. 1990; Xu et al. 1990), SKIP (Zhou et al. 2000), NRARP/5D9 (Krebs et al. 2001; Lamar et al. 2001), and Deltex (Yamamoto et al. 2001). MAM is a glutamine-rich nuclear protein essential for Notch signaling that interacts stoichiometrically with the ICD-CBF1 complex and stabilizes its binding to DNA in vitro (Helms et al. 1999; Wu et al. 2000; Petcherski and Kimble 2000a,b; Kitagawa et al. 2001). Similarly, SKIP can associate with the ICDCBF1 complex and is thought to displace Notch-associated corepressors (Zhou et al. 2000). NRARP is an ankyrin repeat protein that binds to MAM-ICD-CBF1 complexes and promotes Notch proteolysis in Xenopus embryos (Krebs et al. 2001; Lamar et al. 2001). Although Deltex may also function as a transcriptional coactivator (Yamamoto et al. 2001), the presence of WWE and RING finger domains suggests a possible role for this protein in 
the ubiquitination of the Notch receptor as well. Binding of the Notch ICD to CBF1 is mediated by the ANK repeats and $\mathrm{N}$-terminal RAM domain, and a proximal Cterminal region carries the transactivation domain. Several transcriptional coactivators have been proposed to bind the Notch ICD directly, including the histone acetyltransferases PCAF/GCN-5 (Kurooka and Honjo 2000) and CBP/p300 (Oswald et al. 2001), and in Caenorhabditis elegans, the homolog of the Spt6 transcription elongation factor (Hubbard et al. 1996).

The Notch ICD is expressed at very low levels in signaling cells, suggesting that it may undergo rapid proteolytic turnover in the nucleus. The hyperphosphorylated nuclear ICD is recognized through a C-terminal PEST motif by Sel-10, an F-box protein component of a nuclear E3 ubiquitin ligase complex, which marks the ICD for proteolytic degradation (Hubbard et al. 1997; GuptaRossi et al. 2001; Oberg et al. 2001; Wu et al. 2001). The ankyrin repeat protein NRARP can promote turnover of the Notch ICD in embryos; however, its action is independent of the PEST domain (Lamar et al. 2001; E. Lamar and C. Kinter, unpubl.). Similarly, ITCH is a cytoplasmic ubiquitin ligase that promotes ubiquitination of Notch in a PEST-independent manner (Qiu et al. 2000). Thus, ubiquitin-dependent proteolytic events may control Notch signaling at multiple stages (Lai 2002).

Although the events that regulate the turnover of the ICD in the nucleus are not yet fully defined, emerging studies indicate that strong transcriptional activators are frequently marked for destruction by the transcription machinery (for review, see Tansey 2001). For example, the yeast GCN4 activator is phosphorylated by Srb10, a component of the RNA polymerase II-associated Mediator complex, which signals its subsequent destruction by ubiquitin-mediated proteolysis (Chi et al. 2001). Similarly, phosphorylation by the TFIIH CycH-CDK7 complex promotes the turnover of the E2F activator (Vandel and Kouzarides 1999). Recruitment of CBP/p300 appears to connect transcription activation to ubiquitin-mediated proteolysis of the p53 transactivator (Grossman et al. 1998; Zhu et al. 2001). Importantly, ubiquitination of enhancer factors can also be required for transcription activation, as shown recently for the VP16 activation domain (Salghetti et al. 2001), or for exchange of transcriptional coactivator and corepressor complexes (Ostendorff et al. 2002). Moreover, both E3 ubiquitin ligases (Kamura et al. 1998) as well as the 19S regulatory proteasomal complex (Ferdous et al. 2001) have been implicated as functioning directly in the control of RNAPII transcription elongation.

To further characterize the mechanism of transcriptional activation by Notch, we sought to model the activity of the Notch enhancer complex in a chromatinbased, cell-free transcription system. In contrast with current models of Notch function, we find that the activation domain of the ICD is not capable of supporting transcription on its own, and that MAM is an essential component of the minimal functional Notch enhancer. We show that MAM interacts strongly with CBP/p300 and is required for p300-dependent acetylation of nucleo- somes at a minimal Notch enhancer in vitro. Expression of MAM promotes phosphorylation and accumulation of endogenous CBP/p300 proteins in nuclear foci. Moreover, MAM expression strongly enhances the modification and proteolytic degradation of the Notch ICD in the nucleus. The ability of MAM to promote phosphorylation and turnover of the ICD and CBP/p300 depends upon a glutamine-rich C-terminal domain that is required for Notch transcription in vivo. Therefore, MAM may link transcriptional activation with modification, disassembly, and turnover of the Notch enhancer complex in vivo.

\section{Results}

The Notch ICD-CBF1 complex is not sufficient to direct transcription on chromatin in vitro

To reconstitute the Notch enhancer complex on chromatin templates, recombinant human CBF-1 and the human (TAN-1) and Xenopus Notch ICD proteins were expressed in bacteria as fusion proteins with a glutathioneS-transferase (GST) tag and purified by affinity chromatography. The purified proteins were incubated with pNRE (Notch regulatory element), a plasmid containing multiple CBF-binding sites located upstream of a TATA-containing minimal core promoter, in the presence of a Drosophila embryo (S-190) nucleosome-assembly extract and purified Drosophila core histones (Bulger and Kadonaga 1994). Micrococcal nuclease digestion analysis of the chromatin confirmed the presence of a regularly spaced nucleosome array (data not shown) that was capable of repressing core pNRE promoter activity (Fig. 1A, lane 1). In these experiments, transcription was carried out by incubating the chromatin template with a HeLa nuclear extract, and pNRE RNA was detected with a template-specific primer. Transcription from a nonchromatin plasmid containing the $\alpha$-globin promoter $(\alpha$ glo) was included as a control for RNA recovery in the reactions.

As expected, neither CBF1 nor the ICD alone had any effect on pNRE transcription in vitro (Fig. 1A, lanes 2,3). Surprisingly, however, the CBF1-ICD complex was also transcriptionally inactive in this system (Fig. 1A, lane 6). The absence of transcriptional activity was not owing to an inability of the ICD to bind CBF1, because the proteins readily formed a stable ternary complex with DNA in EMSA experiments (Fig. 1B), and the ICD significantly enhanced binding of CBF1 to the nucleosomal pNRE template in DNase I footprint experiments /data not shown). These findings suggest that the Notch ICD, at least in its unmodified state, does not contain an independent activation domain sufficient to initiate transcription on chromatin.

\section{Human MAM provides an activation domain necessary for Notch-regulated transcription in vitro}

Several Notch-specific coactivators have been shown to interact directly with the ICD and may function as an 


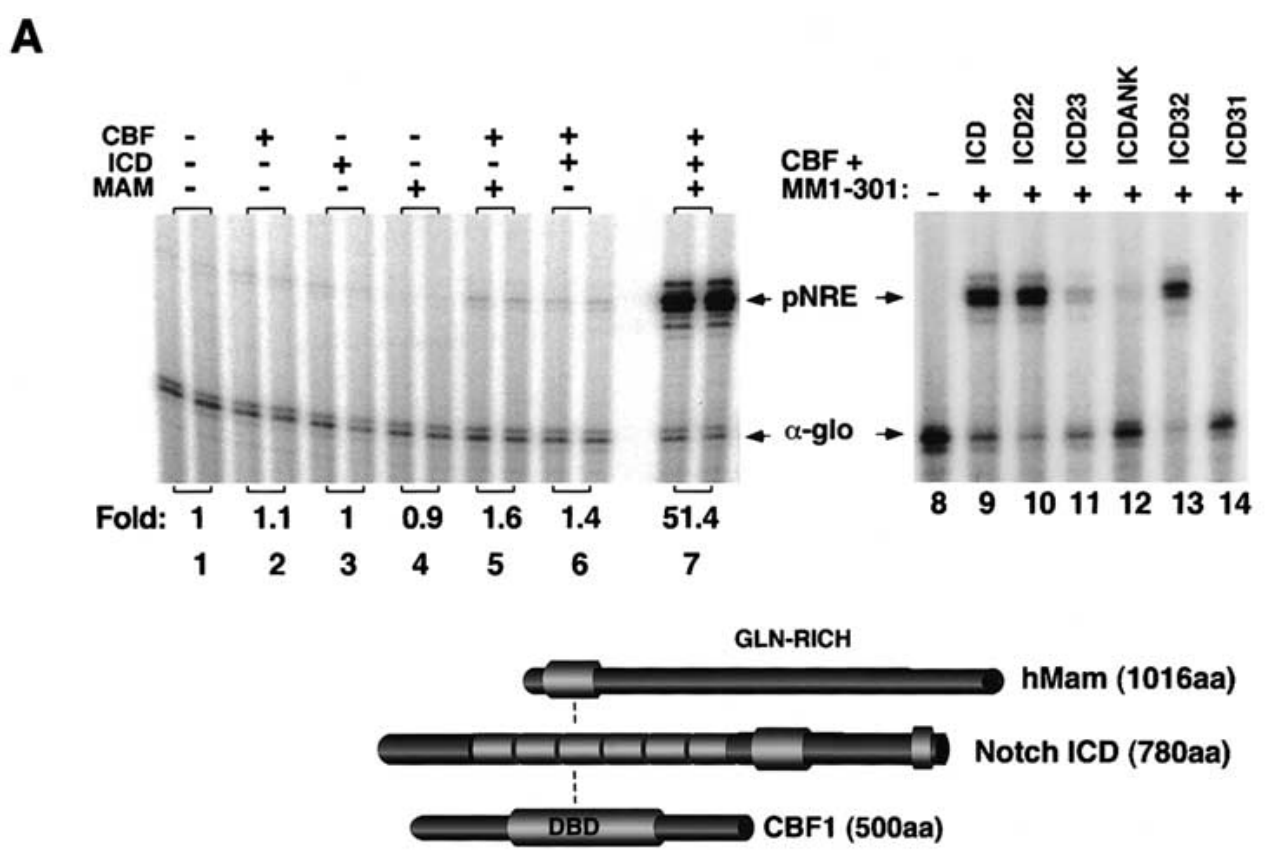

B

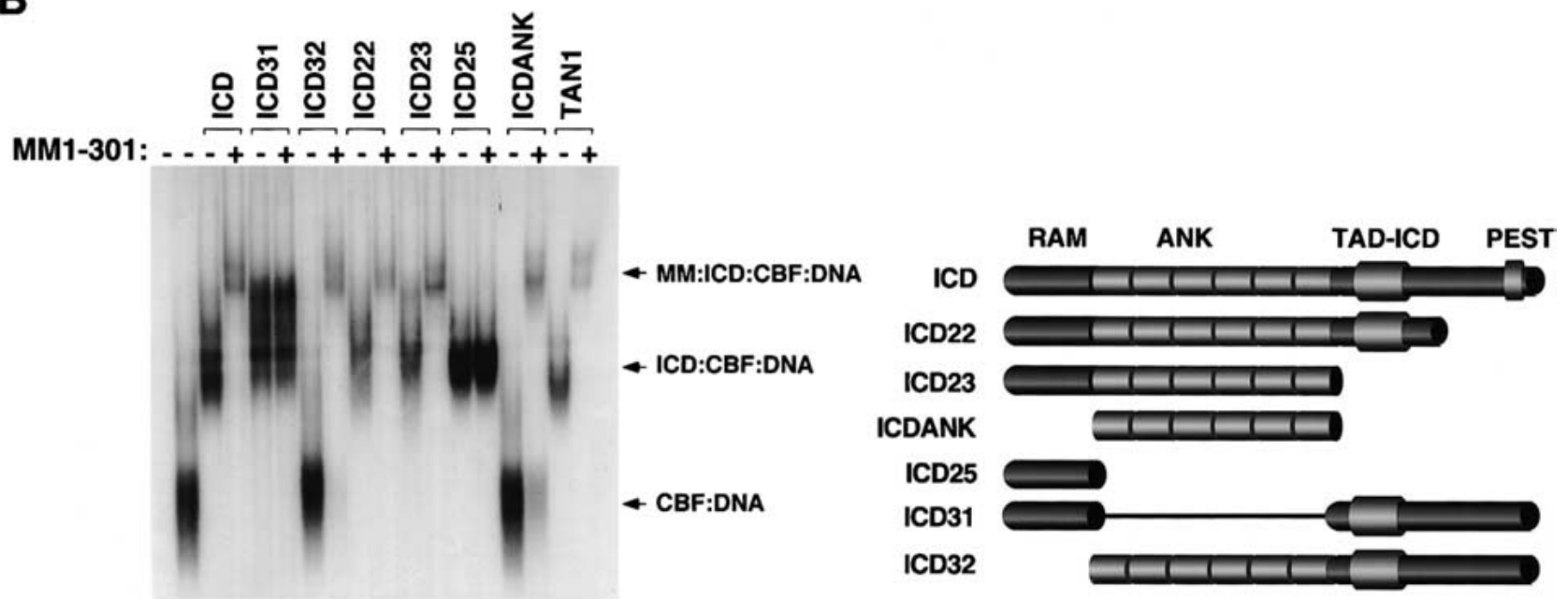

123456789101112131415161718

Figure 1. MAM interacts with and is required for chromatin-dependent transcriptional activation by the Notch (CBF1:ICD) enhancer complex in vitro. (A) Primer-extension analysis of the transcriptional activity of purified recombinant Notch complexes on pNRE chromatin in vitro. Where indicated, the reactions either lacked enhancer factors (lanes 1,8), or contained CBF1 (120 nM, lanes 2,5-7,9-14), the wild-type Xenopus Notch ICD (120 nM, lanes 3,6,7,9), or different mutant ICD proteins (lanes 10-14), and human 1-301MM (120 nM, lanes 4,5,7,9-14). Arrows indicate transcripts originating from the pNRE or control (nonchromatin) $\alpha$-globin ( $\alpha$-glo) templates. The Notch enhancer complex is assembled through binding of the ICD ankyrin repeat (ANK) domain to the central region of CBF1 (amino acids 179-361) and to an N-terminal domain of MAM, as indicated schematically at the bottom of the figure. (B) EMSA analysis of the ability of wild-type and mutant ICD proteins (lanes 3-18, as indicated above each lane) to support a stable ternary complex with CBF1 (lanes 2-18) and MAM (1-301MM; lanes 3,5,7,9,11,13,15) on DNA in vitro. Arrows indicate the various CBF1, ICD-CBF1, and MAM:ICD-CBF1 complexes on DNA. The different mutant ICD proteins tested are depicted to the right of the figure.

integral part of the Notch enhancer complex in vivo. Foremost among these is MAM, which has been shown genetically to be essential for Notch function in many organisms (Xu et al. 1990; Helms et al. 1999; Petcherski and Kimble 2000a,b.; Wu et al. 2000). Consequently we purified the full-length MAM protein using both recombinant baculovirus (MAM1016) and bacterial expression systems (1-1016MM), and we also expressed a C-terminal truncated form of the protein (1-301MM). Interestingly, both the full-length and truncated MAM proteins activated pNRE transcription strongly when incorporated into a complex with the ICD and CBF1 (Fig. 1A, lane 7; see also Fig. 2A). MAM could not activate pNRE transcription on its own (Fig. 1A, lane 4), nor did it enhance 
Fryer et al.

Figure 2. MAM activates pNRE transcription on chromatin in vitro through an $\mathrm{N}$-terminal activation domain (TAD1). (A, left panel) Analysis of the ability of various MAM proteins to activate pNRE transcription in the presence of CBF1 and ICD in vitro. The different MAM truncation or deletion mutants tested are indicated above each lane and are shown schematically at the bottom of the figure. Transcription conditions are as described for Figure 1A. (Right panel) The isolated MAM TAD1 fragment (amino acids 75301) selectively blocks Notch transcription in vitro. Reactions either lacked the Notch enhancer complex (lane 9) or contained the complex (120nM MAM:ICD-CBF1; lanes 10-14) in the absence (lane 10) or presence of 75-301MM (480 nM, lane 11; $960 \mathrm{nM}$, lane 12), a C-terminal fragment of MAM (amino acids 301-1016 fragment, $480 \mathrm{nM}$; lane 13), or the $\beta$-catenin CTARM transactivation domain (960 nM, lane 14). (B, left panel) EMSA analysis of Notch complexes containing wild-type or mutant MAM proteins, as indicated above each lane. Reactions either lacked enhancer factors (lane 1) or contained CBF1 (lanes 2-9), the ICD (lanes 3-9), and various MAM proteins (lanes 4-9), as indicated above each lane. (Right panel) The MAM TAD1 fragment (amino acids 75-301) does not disrupt assembly of the Notch enhancer complex on DNA. The binding reactions either lacked enhancer factors (lane 10) or contained $100 \mathrm{nM}$ each of CBF1 (lanes 11-15), the ICD (lanes 12-15), and 1-1016MM (lanes 14,15), either in the absence (lanes 12,14) or presence (lanes 13,15) of a fivefold excess of 75-301MM. Arrows indicate the different Notch enhancer factor:DNA complexes.

transcription by CBF1 in the absence of the ICD (Fig. 1A, lane 5). Purified MAM bound avidly to the ICD-CBF1DNA complex in EMSA experiments (Fig. 1B), but could not recognize the CBF1-DNA complex in the absence of the ICD (data not shown). MAM did not bind to the pNRE enhancer on its own but modestly enhanced binding of the ICD-CBF1 complex to chromatin in DNase I footprint experiments /data not shown). We conclude that the minimal functional Notch enhancer is a heterotrimer composed of MAM, ICD, and CBF1.

Previous studies have shown that the ANK and RAM regions of the Notch ICD mediate binding to CBF1, whereas the central region contains the transactivation (TAD) and nuclear localization (NLS) domains (Kurooka and Honjo 2000; Oswald et al. 2001). Consequently, we analyzed a series of mutant ICD proteins (Fig. 1A, right panel) to determine which regions of the ICD contribute to Notch transcription in vitro. An ICD mutant lacking the ANK repeats (ICD31) was transcriptionally inactive
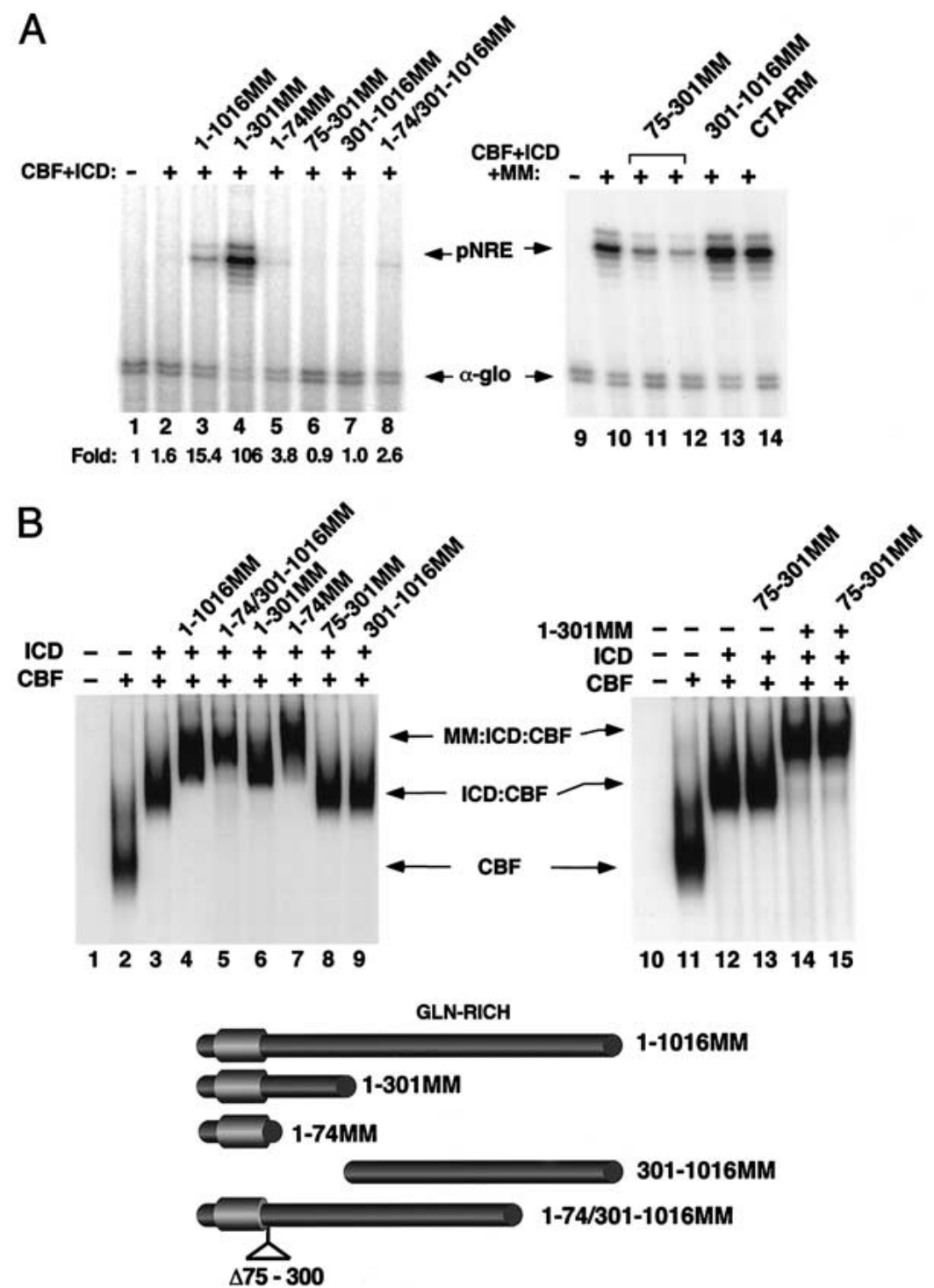

(Fig. 1A, lane 14) and was able to bind CBF1 but unable to form a ternary complex with MAM (Fig. 1B). The C terminus of the ICD was dispensable for pNRE transcription (ICD22; Fig. 1A, lane 10), whereas the TAD/NLS region was required (ICD23; Fig. 1A, lane 11). The ICD23 protein, which lacks the TAD/NLS, was able to bind CBF1 and form a ternary complex with MAM on DNA (Fig. 1B), indicating that its failure to activate Notch transcription was most likely caused by the loss of the activation domain. Removal of the RAM domain (ICD32) disrupted binding to CBF1; however, this mutant retained the ability to form a three-way complex with MAM (Fig. 1B) and was able to support Notch transcription in vitro (Fig. 1A, lane 13). A protein containing only the ANK repeats (ICDANK) failed to bind CBF1 (Fig. 1B) but could weakly support a three-way complex with MAM (Fig. 1B). The ICDANK protein was transcriptionally inactive (Fig. 1A, lane 12), but the transcriptional defect of this mutant as well as that of the ICD23 mutant could be overcome by using higher levels of the 
protein (data not shown). From these data, we conclude that the primary role of the ICD is to tether MAM to CBF1 through the ANK repeats, but that the TAD/NLS region of the ICD also contributes to Notch transcription in a MAM-dependent manner.

\section{The central MAM transactivation domain (TAD1) interacts with $C B P / p 300$}

These findings suggest that MAM provides an activation domain necessary for initiation of Notch transcription on chromatin in vitro. We found that a C-terminal truncated form of MAM (1-301MM) activated pNRE transcription even more strongly than wild-type MAM (11016MM; Fig. 2A, cf. lanes 3 and 4), suggesting that the activation domain is contained within the first third of the MAM protein. Further analysis indicated that two MAM mutants lacking the ICD-binding domain at the $\mathrm{N}$ terminus were transcriptionally inactive (Fig. 2B, lanes 6 and 7), and unable to bind to the ICD in EMSA experiments (Fig. 2B, lanes 8 and 9), confirming that MAM must interact with the ICD to activate Notch transcription in vitro. An N-terminal fragment of MAM that was able to bind the ICD (Fig. 2B, lane 7) was also found to be transcriptionally inactive in vitro (1-74MM; Fig. 2A, lane 5), indicating that the MAM activation domain (TAD1) lies between amino acids 75 and 301. Consistent with this possibility, a full-length MAM protein lacking TAD1 (1-74/301-1016MM) was significantly impaired in its ability to activate pNRE transcription (Fig. 2B, lane 8 ), even though it could assemble into the Notch enhancer complex (Fig. 2B, lane 5). Moreover, the isolated TAD1 fragment (75-301MM) selectively blocked pNRE transcription in vitro when incubated at levels four- to eightfold higher than that of the ICD (Fig. 2A, cf. lane 10 with lanes 11 and 12). In contrast, pNRE transcription was unaffected by equivalent levels of either a C-terminal fragment of MAM (amino acids 301-1016; Fig. 2A, lane 13 ) or the CT-ARM fragment of the $\beta$-catenin ( $\beta$-cat) transcription factor (Fig. 2A, lane 14; Tutter et al. 2001). The inhibition by 75-301MM was specific because it did not affect transcription in vitro by the unrelated LEF-1$\beta$-catenin enhancer complex (data not shown), nor did it disrupt the assembly of the Notch enhancer complex in EMSA experiments (Fig. 2B, lanes 14,15).

To determine whether the 75-301MM fragment was acting to sequester Notch coactivators, we examined the HeLa nuclear proteins that interact with TAD1 using a GST protein affinity-selection (pull-down) approach. The bead-coupled 75-301MM protein was incubated with a crude HeLa nuclear extract, and the associated proteins that remained following stringent washing were eluted by boiling and visualized by SDS-PAGE and silver-staining. As shown in Figure 3A, the $75-301 M M$ protein bound two prominent HeLa nuclear proteins of $300 \mathrm{kD}$ and $100 \mathrm{kD}$ (Fig. 3A, lane 3). Neither of these proteins was present in eluates from 75-301MM beads that had not been incubated with the HeLa nuclear extract (Fig. $3 \mathrm{~A}$, lane 2). The 300-kD protein was subjected to analysis by MALDI-TOF mass spectrometry and identified as the histone acetyltransferase, CBP/p300. The identification was confirmed by Western blot analysis, which revealed that p300 interacts strongly with full-length $1-1016 \mathrm{MM}$ as well as 75-301MM proteins (Fig. 3B, lanes 6 and 7, respectively), but not with $1-74 M M$ or GST alone (Fig. 3B, lanes 2 and 8 , respectively). Removal of the 75-301aa domain greatly reduced binding to p300 (Fig. 3B, cf. lanes 5 and 6), indicating that MAM does not contain other high-affinity interaction sites for p300. Although it has been reported that the Notch ICD interacts directly with p300 (Oswald et al. 2001), we failed to detect any interaction between p300 and GST-ICD by Western blot under the stringent conditions used here (data not shown), and conclude that MAM, not the ICD, is the preferred target for p300 in the Notch enhancer.

To assess whether p300 functions as a positive cofactor for Notch in vitro, CBF1 and the ICD were incubated with the template during nucleosome assembly, and MAM and recombinant p300 were added at a later step, following chromatin assembly but prior to transcription. Transcription of the pNRE template by the Notch enhancer complex was relatively inefficient under these conditions (Fig. 3C, lane 6), but was strongly activated by purified p300 (Fig. 3C, lane 7). As expected, p300 was transcriptionally inactive in the absence of the Notch enhancer factors (Fig. 3C, lanes 2-4). Activation of the Notch enhancer complex by p300 was blocked by LysCoA, a selective inhibitor of the histone acetyltransferase activity of p300 (Lau et al. 2000; data not shown), indicating that acetylation is important for p300 function in this system.

Although p300 can readily acetylate free histones in vitro, acetylation of assembled nucleosomal arrays has been shown to require an activator-mediated targeting step (Ito et al. 2000). Consequently, we asked whether the Notch enhancer factors could recruit p300 to acetylate the nucleosomal pNRE template. For this experiment, the enhancer proteins were allowed to bind the template during nucleosome assembly, and the chromatin template was purified prior to incubation with recombinant p300. Acetylation of pNRE nucleosomal histones was measured by incorporation of ${ }^{3} \mathrm{H}$-acetyl CoA. As shown in Figure 3D, p300 was unable to acetylate pNRE chromatin templates that had been assembled either in the absence of enhancer factors (Fig. 3D, lane 1) or in the presence of the ICD-CBF1 complex (Fig. 3D, lane 2). However, p300 was able to access the pNRE template in the presence of Notch enhancer complexes containing either full-length MAM (Fig. 3D, lane 3) or 1-301MM (Fig. 3D, lane 4). As expected, p300-mediated acetylation of the template also required CBF1 and the Notch ICD (Fig. 3D, lane 5). Importantly, the TAD1 region of MAM was required for p300-directed histone acetylation (Fig. $3 \mathrm{D}$, lane 6), and acetylation could be competed in trans by the 75-301MM fragment (data not shown). The shorter MAM protein 1-301MM was more efficient than wild-type MAM at supporting acetylation by p300, consistent with its higher transcriptional activity in vitro. Taken together, these data indicate that MAM recruits p300 to acetylate pNRE nucleosomal histones, and that 
Fryer et al.

Figure 3. MAM interacts specifically with nuclear CBP/p300 and promotes p300-mediated nucleosome acetylation in vitro. $(A)$ SDS-PAGE analysis of HeLa nuclear proteins that interact with the MAM TAD1 fragment. A crude HeLa nuclear extract was incubated with either glutathione beads (lane 1) or GSTMAMTAD1 beads (lane 3), and the associated proteins were eluted by boiling and analyzed by SDS-PAGE and silver staining. The arrow indicates a band identified as CBP/p300 through tryptic digestion and MALDI-TOF mass-spectrometry analysis. (B) HeLa nuclear extract was incubated with either GST (lane 8) or various GST-MAM protein-coupled beads (lanes 2-7) as indicated above each lane, and analyzed by immunoblotting for associated nuclear p300 protein. The input nuclear p300 protein is shown in lane 1. (C) Purified recombinant p300 enhances Notch transcription in vitro. Transcription reactions contained the pNRE template incubated in the absence of enhancer factors (lane 1,5) or with the Notch enhancer complex (MAM-ICD$\mathrm{CBF} 1$; lanes 6,7), which was added following chromatin assembly. Where indicated, recombinant p300 was incubated with the chromatin template for $30 \mathrm{~min}$ prior to analysis of transcription by primer extension (lanes 2-4,7). (D) Purified p300 promotes acetylation of pNRE nucleosomal histones in a MAM-dependent manner in vitro. The pNRE chromatin assembly reactions either lacked enhancer factors (lane 1), or contained CBF1-ICD (lane 2-4,6) and wild-type or mutant MAM protein (lanes 3-6), as indicated above the lanes. The pNRE template was purified following chromatin assembly and incubated with recombinant p300 and ${ }^{14} \mathrm{C}$-acetyl CoA, and the labeled nucleosomal histones were identified by SDS-PAGE and fluorography.

TAD1 likely blocks Notch transcription by disrupting the binding of p300 to MAM.

Overall these findings were surprising, because a MAM truncation mutant similar to 1-301MM has been shown previously to block Notch signaling in vivo (Wu et al. 2000). We interpreted these results to suggest that MAM may contain two distinct transcription activation domains: (1) a previously unrecognized central activation domain (TAD1; amino acids 75-301); and (2) a Cterminal activation domain (TAD2) that is essential for Notch activity in vivo but does not contribute to Notch transcription in vitro under the conditions tested here. To investigate this possibility further, we analyzed whether both TAD1 and TAD2 are required for Notch signaling in vivo, and whether either domain influences the subnuclear localization of MAM, in the experiments described below.

\section{A mutant MAM unable to bind CBP/p300 functions as a dominant-negative inhibitor of Notch signaling in Xenopus embryos}

To determine whether the p300 interaction domain (TAD1; amino acids 75-301) is required for MAM function in vivo, Xenopus embryos were injected with RNA encoding either the wild-type or mutant MAM proteins, and analyzed for Notch function by scoring the number of primary neurons that form in the neural plate (Lamar et al. 2001). In this assay, increased Notch signaling reduces the number of differentiated cells (primary neurons) that form, as visualized by staining for a neuralspecific form of tubulin, whereas disabled Notch signaling results in an increased number of neurons. Injection of $\Delta 74-301 \mathrm{MM}$ RNA markedly increased the number of neurons formed relative to the uninjected control half of the embryo (Fig. 4), indicating that Notch signaling is blocked in vivo by a MAM mutant protein that lacks TAD1. Consistent with a previous report $(\mathrm{Wu}$ et al. 2000), we also found that $1-301 M M$ is a dominant-negative inhibitor of Notch signaling in Xenopus embryos (Fig. 4). Both $\Delta 74-301 M M$ and 1-301MM also blocked the induction by the ICD of two primary Notch target genes, ESR1 and ESR7, in animal cap experiments (Lamar et al. 2001; data not shown), confirming that the defect in Notch signaling was transcriptional. Moreover, neither of the MAM mutant proteins was able to enhance ICD-dependent Notch transcription in transient expression experiments (data not shown). We conclude that both TAD1 (amino acids 75-301) and the C-terminal activation domain (TAD2; amino acids 301-1016) are required for MAM-dependent activation of Notch target and reporter genes in vivo, whereas the initiation of Notch transcription on chromatin in vitro requires the MAM TAD1, but not TAD2, domain. 


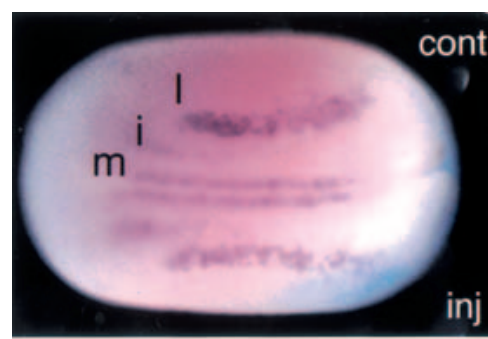

\section{HMM Full Length}

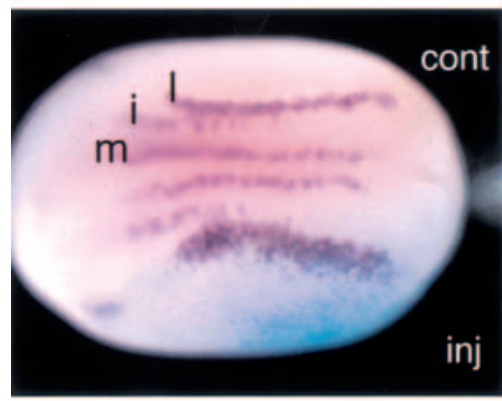

$\mathrm{HMM}$ $\Delta 74-301$

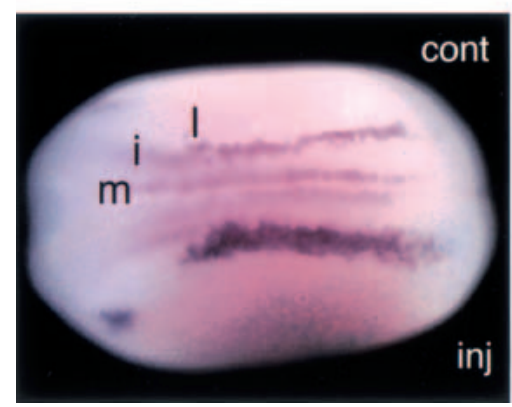

HMM

Figure 4. Both TAD1 and the C-terminal activation domain (TAD2) of MAM are required for Notch signaling in injected Xenopus embryos. Xenopus embryos at the two-cell stage were injected with RNA encoding wild-type (Full-length) or mutant human MAM proteins (HMM 4 4-301; HMM1-301) along with lacZ RNA as a tracer. The embryos were fixed and stained for $\beta$-galactosidase expression and double-labeled by whole-mount in situ hybridization for a neural-specific tubulin. Notch signaling was assessed by counting the number of primary neurons on the neural plate for the half of the embryo that was injected (inj) relative to that for the uninjected control (cont). (m) Midline; (i) interneuron; (1) lateral neuron.

\section{Expression of MAM directs CBP/p300 to nuclear foci in vivo}

To investigate whether the failure of the 1-301MM protein to support Notch signaling in vivo might be owing to an inability to localize properly to the nucleus, or to altered subnuclear localization of MAM within PML bodies (Wu et al. 2000), we examined the subcellular distribution of transiently expressed Myc-tagged wildtype and truncated MAM proteins by indirect immunofluorescence assay (IFA) and deconvolution microscopy. When visualized by IFA, both wild-type MAM and 1-301MM were found to localize to the nucleus of transfected cells (Fig. 5). Overexpressed full-length MAM has been shown previously to localize to PML bodies (Wu et al. 2000); however, in our experiments in HeLa cells most of the expressed MAM was distributed homoge- neously throughout the nucleus. In cells expressing fulllength MAM, the PML bodies were enlarged but still did not colocalize with MAM (data not shown). Unexpectedly, we noticed that expression of full-length MAM induces the relocalization of endogenous p300 and CBP proteins to nuclear foci (Fig. 5). In transfected cells the CBP and p300 proteins were depleted from the nuclei and accumulated in these structures up to $72 \mathrm{~h}$ posttransfection. The aggregate size varied dramatically in different cells from multiple small foci to larger structures of 1-10 $\mu \mathrm{m}$. Surprisingly, these foci do not appear toxic to the cells, at least over a period of $72 \mathrm{~h}$ following transfection. Identical structures were observed upon expression of MAM in MCF7 and 293 cells (data not shown), indicating that formation of these nuclear foci is not cell-type-specific. This effect was relatively specific for CBP and p300 because MAM did not affect the nuclear distribution of unrelated proteins such as the splicing factor sc-35 (data not shown).

We next asked whether the $\mathrm{CBP} / \mathrm{p} 300$ foci might arise as a consequence of altered Notch signaling. The CBP/ p300-containing structures did not form upon overexpression of the Notch ICD or CBF1 proteins (data not shown), indicating that these structures do not reflect aberrant Notch signaling, nor were foci observed upon expression of other strong transcriptional activators such as $\beta$-catenin and HIV-1 Tat protein (data not shown). We conclude that overexpression of components of the Notch pathway is not sufficient to alter $\mathrm{CBP} / \mathrm{p} 300$ localization. Interestingly, expression of the 1-301MM protein did not cause the endogenous $\mathrm{CBP} / \mathrm{p} 300$ proteins to accumulate in nuclear foci (Fig. 5), indicating that this phenomenon requires the TAD2 region of MAM. Because the 1-301MM protein contains the high-affinity binding site for $\mathrm{CBP} / \mathrm{p} 300$, we conclude that binding of $\mathrm{CBP} / \mathrm{p} 300$ to MAM is not sufficient to redirect its subnuclear localization, consistent with the observation that MAM does not significantly colocalize with CBP/ p300 in the foci under these conditions. Therefore, these structures may result from Notch-independent effects of MAM on the expression of cellular genes, or from the direct action of MAM when overexpressed in the nucleus.

\section{Coexpression with MAM and CBF1 enhances the modification and proteolytic turnover of the Notch ICD in vivo}

In further studies, we found that coexpression of MAM and CBF1 with the Notch ICD failed to induce any of the Notch enhancer components to localize in the CBP/p300 foci (data not shown). Interestingly, however, we noticed that coexpression of MAM and CBF1 had a striking effect on the apparent stability of the Notch ICD in transiently transfected cells. In the experiment shown in Figure 6, Myc-tagged MAM, CBF1, and ICD proteins were expressed together in different combinations in HeLa or $\mathrm{C} 2 \mathrm{C} 12$ cells and analyzed by immunoblotting $48 \mathrm{~h}$ after transfection. Although the Notch ICD (ICD11) protein was readily expressed on its own or in the presence of 
Fryer et al.

\section{(A) 1-1016MM}
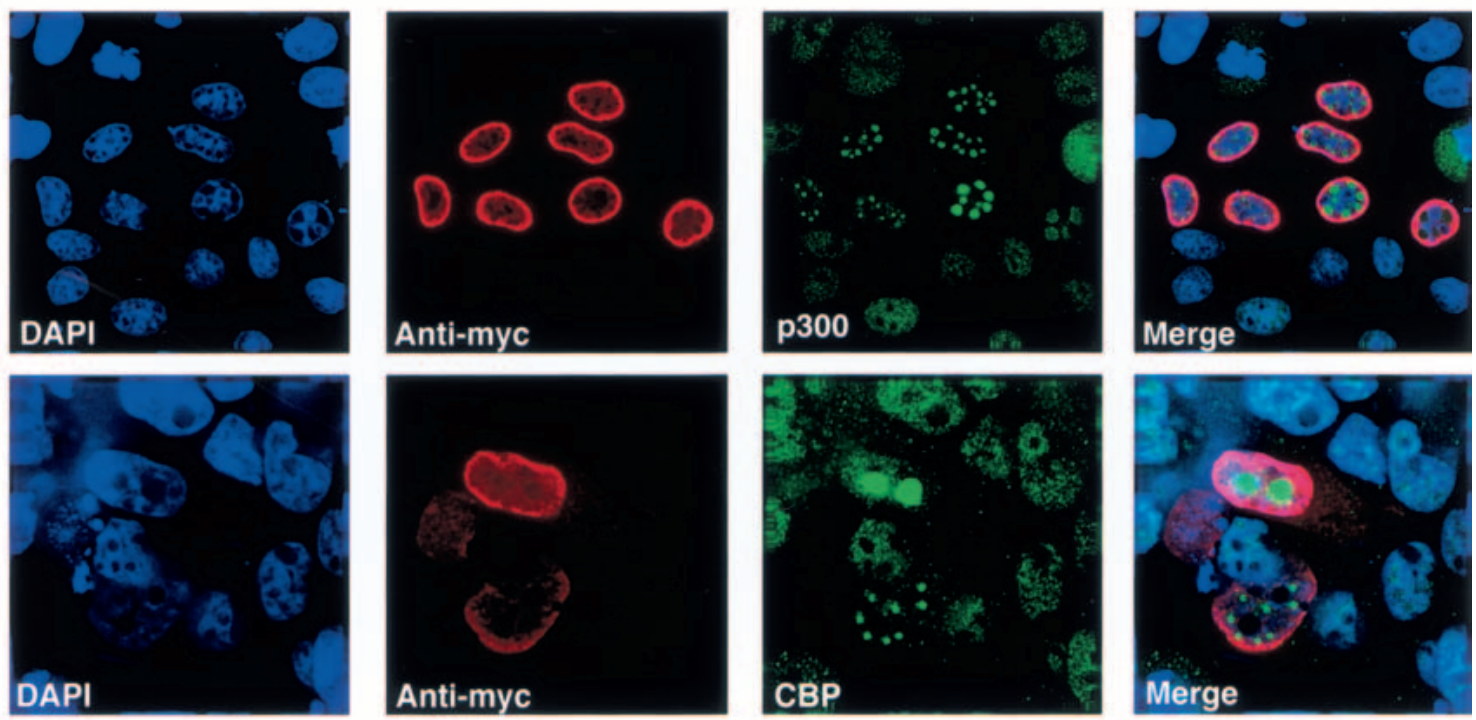

\section{(B) 1-301 MM}
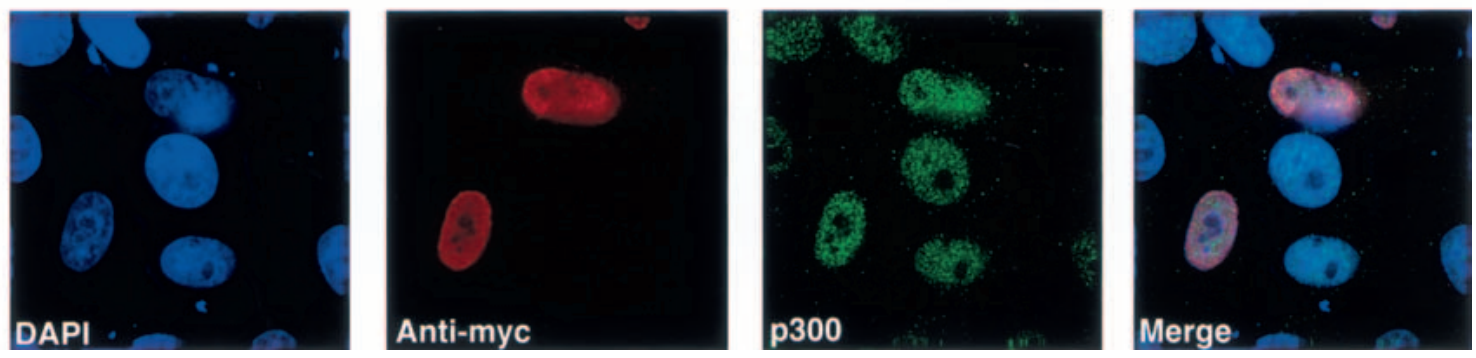

Figure 5. Human MAM directs endogeneous CBP/p300 to nuclear foci in vivo in a TAD2-dependent manner. Transiently expressed Myc-tagged wild-type (1-1016MM) or mutant MAM proteins (1-301MM) and endogenous p300 and CBP proteins were visualized by indirect immunofluorescence and deconvolution microscopy in HeLa cells. (A) HeLa cells transiently transfected with 1016MM-Myc were imaged for Myc immunofluorescence (red) and endogenous p300 (upper panel) or CBP (lower panel) immunofluorescence (green). Cells were counterstained with DAPI (blue) to show the position of the nucleus for all the cells in the field. $(B)$ HeLa cells were transiently transfected with 1-301MM-Myc and analyzed as in $A$.

CBF1, only very low levels of the ICD accumulated when coexpressed with MAM and CBF1 (Fig. 6A, cf. lanes 1 and 3). In contrast, a mutant form of the ICD lacking the PEST domain (ICD22) was stable in the presence of MAM and CBF1. The apparent decreased stability of the ICD was most pronounced when all three Notch enhancer proteins were expressed. Thus, the ICD was stable when expressed with MAM (Fig. 6B, cf. lanes 2 and 4), but not with MAM and CBF1 (Fig. 6B, cf. lanes 6 and 8). Although the Notch cofactor NRARP influences the stability of Notch in Xenopus embryos (Lamar et al. 2001), coexpression of NRARP (N) had no effect on the apparent stability of any of the Notch enhancer components in these experiments (Fig. 6A,B). Interestingly, the Notch ICD was stable when coexpressed with CBF1 and the C-terminally truncated MAM (1-301MM) protein, which lacks the TAD2 domain (Fig. 6C, cf. lanes 2 and 5).

In experiments where low levels of the Notch ICD could be detected in the presence of MAM and CBF1, the ICD was found to migrate on SDS-PAGE gels as a modified form that resembles the hyperphosphorylated form (Fig. 6C, cf. lanes 9 and 10). In contrast, no evidence of hyperphosphorylation was observed when the ICD was coexpressed with $\mathrm{CBF} 1$ and the shorter MAM (1-301MM) protein (Fig. 6C, cf. lanes 9-11). Further analysis revealed that modification of the ICD by MAM was reversed upon treatment with protein phosphatase (Fig. 7A, cf. lanes 4 and 5), but not with Vanadate-inactivated phosphatase (Fig. 7A, lane 6). We conclude that MAM promotes the hyperphosphorylation and proteolytic turnover of the Notch ICD in the presence of CBF1. Interestingly, Western blot analyses also revealed a change in the migration of endogenous CBP in cells expressing MAM (Fig. 7B, cf. lanes 2 and 3). This altered migration was reversed by treatment of the cells with protein phosphatase in a manner that could be inactivated with Vanadate (Fig. 7B, cf. lanes 7 and 8), indicat- 

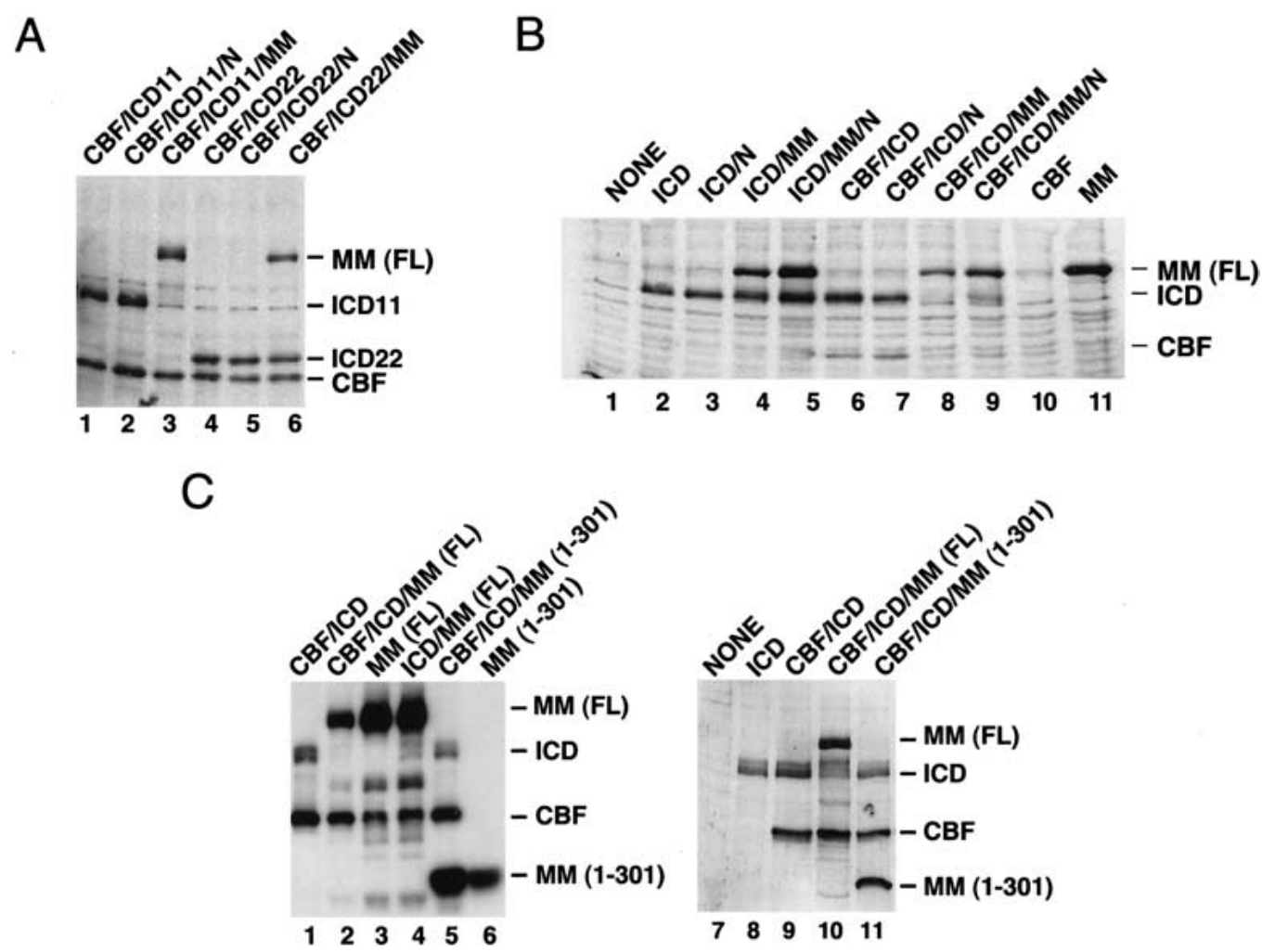

Figure 6. The MAM TAD2 region promotes the modification and turnover of Notch ICD in vivo. (A) MAM and CBF1 enhance the PEST-dependent proteolytic turnover of the Notch ICD in vivo. The expression of Myc-tagged Notch enhancer proteins in C2C12 cells was detected $48 \mathrm{~h}$ after transfection by SDS-PAGE of cell extracts and immunoblotting with an anti-Myc antibody. The expressed proteins included CBF1 (lanes 1-6), full-length ICD (ICD11; lanes 1-3), a C-terminal truncated ICD lacking the PEST domain (ICD22, lanes 4-6), full-length MAM (1-1016MM; lanes 3-6), and the Notch-specific factor NRARP (N, lanes 2,5). (B) MAM-dependent turnover of the Notch ICD requires CBF1 in vivo. Full-length Notch ICD (ICD; lanes 2-9), MAM (MM; lanes 4,5,8,9,11), CBF1 (lanes 6-10), and NRARP (lanes 3,5,7,9) were expressed in HeLa cells and analyzed for protein expression $48 \mathrm{~h}$ after transfection in vivo. (C) HeLa cells (left panel) or C2C12 cells (right panel) were transfected with CBF1 (lanes 1,2,5,9-11), ICD (lanes 1,2,4,5,8-11), and either full-length (FL; lanes 2-4,10) or 1-301MM (1-301; lanes 5,6,11), and protein levels were examined as in A. The MAM-dependent modification of the ICD is evidenced by its altered mobility by SDS-PAGE (lane 10).

ing that MAM also promotes phosphorylation of CBP in vivo. This change in CBP phosphorylation was not observed upon expression with the C-terminal truncated MAM protein (1-301MM), indicating that TAD2 is required for MAM to promote phosphorylation of endogenous CBP in vivo. Taken together, these findings suggest that assembly of MAM into the Notch enhancer complex promotes the phosphorylation of CBP as well as the phosphorylation and turnover of the associated Notch ICD. These findings are summarized in the model shown in Figure 8, and discussed further below.

\section{Discussion}

\section{A key role for MAM in the Notch enhancer complex}

A common feature of developmental signaling pathways such as Notch, Wnt/Wg, Hedgehog, and TGF- $\beta$ is the ligand-dependent mobilization of dedicated coactivators that target specific classes of enhancer factors. Like these other induced coactivators, the intracellular domain of the Notch receptor carries a potent transactivation do- main that is otherwise lacking in its DNA-binding partner, CBF1 (Artavanis-Tsakonas et al. 1999; Mumm and Kopan 2000). Through reconstituting the activity of the Notch enhancer complex on chromatin, we find that the Notch ICD functions primarily to connect CBF1 to a second non-DNA-binding coactivator, MAM. In addition, we find that the activation domain of the ICD functions in a MAM-dependent manner in vitro. These observations strongly suggest that the minimal functional Notch enhancer complex is a heterotrimer composed of MAM, the Notch ICD, and CBF1.

In this respect, the Notch transactivation mechanism differs from that used by the Wnt/Wg signaling pathway, because the Wnt-responsive $\beta$-catenin-LEF-1 enhancer complex, unlike the ICD-CBF1 complex, is sufficient to initiate transcription on chromatin templates in vitro (Tutter et al. 2001). A key role for Notch in the assembly of multisubunit signaling complexes might serve to modulate the response of different cells to Notch ligand, depending on the level of available MAM in the nucleus. Moreover, the ICD may assemble multiple types of complexes with different coactivators such as SKIP (Zhou et 
Fryer et al.
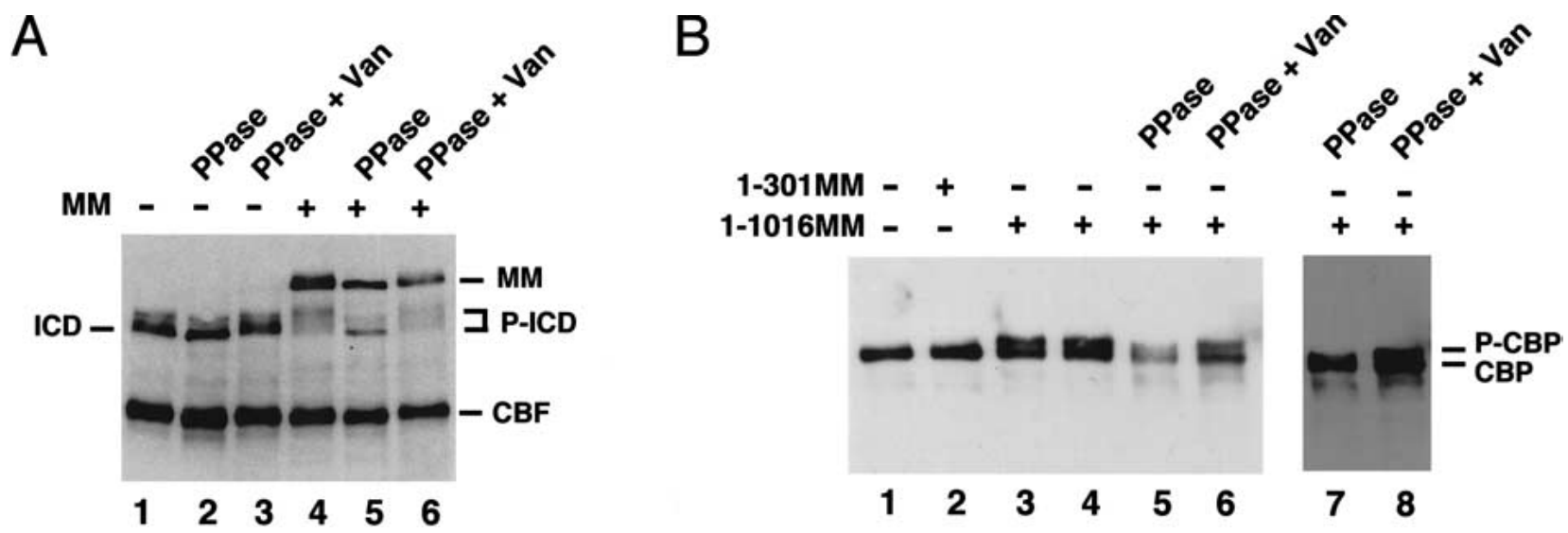

Figure 7. Transient expression of MAM alters the phosphorylation of the Notch ICD and endogenous CBP proteins. (A) C2C12 cells were transfected with myc-tagged forms of ICD and CBF1 in the absence (lanes 1-3) or presence (lanes 4-6) of MAM, and cell extracts prepared $48 \mathrm{~h}$ after transfection were immunoprecipitated with an anti-myc antibody (9E10). The isolated proteins were resuspended in phosphatase buffer (lanes 1,4), phosphatase buffer containing $0.2 \mathrm{U}$ of acid phosphatase (lanes 2,5), or phosphatase buffer containing $0.2 \mathrm{U}$ of acid phosphatase plus $1 \mathrm{mM}$ sodium orthovanadate (Van; lanes 3,6). Immunoprecipitated complexes were detected by Western blotting with an anti-myc antibody. (B) 293T cells were transfected with pCDNA empty vector (lane 1), 1-301MM (lane 2), or 1-1016MM (lanes 3-6), and cell extracts prepared $48 \mathrm{~h}$ after transfection were immunoprecipitated with an anti-CBP antibody. The isolated complexes were untreated (lanes 1-3) or resuspended in phosphatase buffer (lane 4), phosphatase buffer containing $0.2 \mathrm{U}$ of acid phosphatase (lane 5), or phosphatase buffer containing $0.2 \mathrm{U}$ of acid phosphatase plus $1 \mathrm{mM}$ sodium orthovanadate (Van; lane 6). The immunoprecipitated complexes were detected by Western blotting with an anti-CBP antibody. Lanes 7 and 8 show a longer exposure of the reactions shown in lanes 5 and 6.

al. 2000), which might functionally substitute for MAM in some cells. Previous studies have shown that binding of the Notch ICD to yet a different potential coactivator, Deltex, excludes binding to CBF1 (Yamamoto et al. 2001). Thus, an ICD-Deltex complex might target other enhancer-binding proteins and activate a distinct set of genes in response to Notch signaling. Although the composition of individual Notch signaling complexes has not yet been well defined, our data indicate that displacement of $\mathrm{CBF}$-associated corepressors by a heterodimeric ICD-CBF1 complex would not be sufficient to activate a Notch target gene in chromatin (Fig. 8A).

Previous studies have shown that MAM enters the Notch enhancer complex through binding to the ICD, in a step that also requires CBF1 (Fig. 8B; Petcherski and Kimble 2000b). Our findings suggest that MAM carries two distinct transactivation domains (TAD1, TAD2) that act in concert with the ICD activation domain. In vitro, MAM acts through TAD1 to recruit $\mathrm{CBP} / \mathrm{p} 300$ to the Notch enhancer complex (Fig. 8B) and direct acetylation of the nucleosomal pNRE template. Although a recent report indicates that the ICD can bind directly to p300 (Oswald et al. 2001), we show that MAM is essential for the ICD-CBF1 complex to recruit p300 to the template in vitro (Fig. 3D). One possibility is that the ICD-TAD may help stabilize the association of p300 with the enhancer complex after it has been recruited by MAM. It is interesting to note that the MAM C-terminal activation domain (TAD2) does not contribute to transcription in the system used here, which monitors only the steps leading to initiation of transcription. Consequently, it will be important to assess whether the MAM TAD2 controls a postinitiation step, such as promoter clearance or elongation, or whether the action of TAD2 requires additional coactivators in the complex, or posttranslational modification of the Notch enhancer proteins.

\section{MAM expression induces phosphorylation} and accumulation of CBP/p300 in nuclear foci

Unexpectedly, we find that expression of MAM induces endogenous $\mathrm{CBP} / \mathrm{p} 300$ proteins to accumulate in multiple nuclear foci in vivo (Fig. 5). We also show that these structures do not form upon expression of a mutant MAM protein lacking the C-terminal TAD2 region (1$301 \mathrm{MM})$. Thus, binding of MAM to $\mathrm{CBP} / \mathrm{p} 300$, which is mediated through TAD1, is not sufficient to cause CBP/ p300 to accumulate in these structures. Expression of other Notch components (ICD, CBF1) did not affect the subnuclear localization of $\mathrm{CBP} / \mathrm{p} 300$, indicating that these foci are not a consequence of high levels of Notch signaling in the nucleus. One possibility is that MAM may regulate the expression or modification of $\mathrm{CBP} /$ p300 independently of Notch signaling. Indeed, we find that the MAM-induced foci are accompanied by increased phosphorylation of CBP, and this phosphorylation requires the C-terminal TAD2 domain of MAM (Fig. 7). Consequently, overexpression of MAM in the nucleus may promote widespread phosphorylation of CBP, which may cause the $\mathrm{CBP} / \mathrm{p} 300$ proteins to concentrate in these structures. Changes in $\mathrm{CBP} / \mathrm{p} 300$ phosphorylation have been shown to alter its activity and differentially affect its interactions with other transcription factors (Ait-SiAli et al. 1998). It will therefore be important to assess whether MAM promotes $\mathrm{CBP} / \mathrm{p} 300$ phosphorylation 


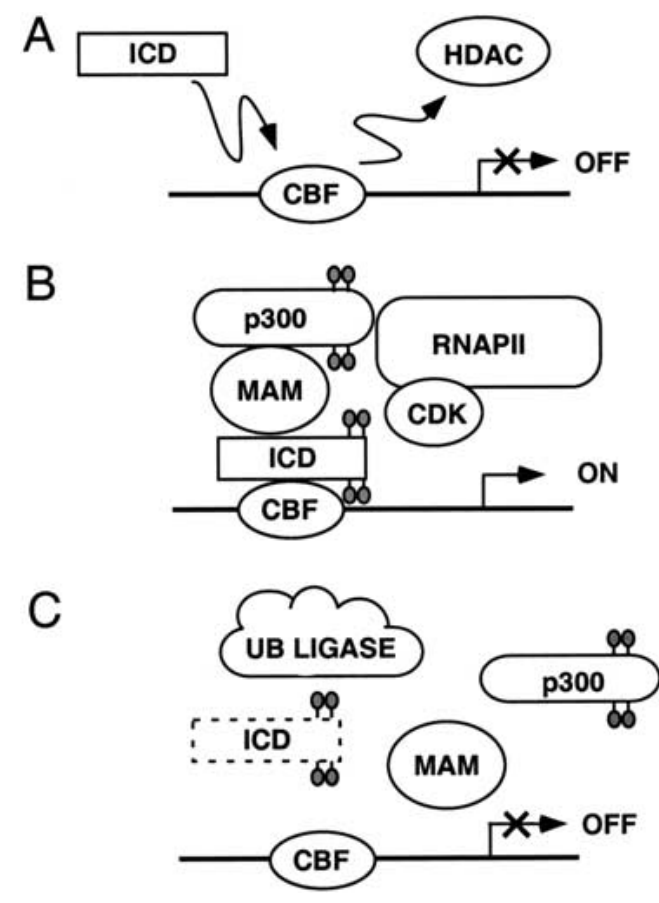

Figure 8. Model for the mechanism of Notch transcription. $(A)$ Notch target genes are repressed through CBF1 complexes that contain histone deacetylases (HDAC) and other corepressors (Dou et al. 1994; Kao et al. 1998; Hsieh et al. 1999; Morel et al. 2001). Although previous studies have shown that binding of the Notch ICD to CBF1 can displace corepressor complexes (Kao et al. 1998; Zhou et al. 2000), the data presented here indicate that a CBF1-ICD complex would be insufficient to activate Notch transcription in the absence of MAM. (B) A threeway complex containing CBF1, ICD, and MAM is required for Notch transcription on chromatin templates in vitro. $\mathrm{CBP} / \mathrm{p} 300$ is recruited through the MAM TAD1 region to promote transcription initiation and acetylate nearby nucleosomal histones. The ICD activation domain also contributes to transcription at this step. We find that MAM induces the phosphorylation of both $\mathrm{CBP} / \mathrm{p} 300$ and the Notch ICD in a step that requires the MAM TAD2 region. Phosphorylation of the Notch ICD by MAM also requires CBF1, which may stabilize binding of MAM to the ICD (Petcherski and Kimble 2000b). Widespread phosphorylation of $\mathrm{CBP} / \mathrm{p} 300$ proteins by MAM may contribute to the accumulation of these proteins in nuclear foci. It remains to be determined whether the MAM-induced phosphorylation events might be carried out by cyclin-dependent kinases associated with the RNA polymerase II (RNAPII) complex (Price 2000; Orphanides and Reinberg 2002) or other protein kinases associated with MAM. $(C)$ The phosphorylated ICD may be targeted for ubiquitination by ubiquitin ligase complexes, leading to disassembly of the enhancer complex and proteolytic degradation of the ICD.

within the Notch enhancer complex (Fig. 8B), and whether phosphorylation of $\mathrm{CBP} / \mathrm{p} 300$ is important for transcriptional activation by Notch.

\section{The MAM C-terminal activation domain couples} transcription activation with turnover of the ICD

The timing of Notch signaling is tightly controlled in developmental processes such as somite formation, dur- ing which Notch target genes such as cHairy1 and mHES1 undergo periodic cycles of expression at the direction of a molecular oscillator, or vertebrate segmentation clock (for review, see Pourquie 1999). This clock may be established through the intrinsic timing of Notch signaling as well as the half-life of Notch-induced transcriptional repressors. Previous studies have established that the Notch ICD is subject to proteolytic degradation in the nucleus through the action of the ubiquitin ligases such as Sel-10 (Hubbard et al. 1997; GuptaRossi et al. 2001; Oberg et al. 2001; Wu et al. 2001). Rapid turnover of the ICD may be required to allow genes to respond rapidly to subsequent cycles of Notch signaling. We find that coexpression with MAM and CBF1 promotes the phosphorylation and proteolytic turnover of the ICD in vivo (Fig. 6), indicating that MAM couples transcription activation with degradation of the ICD. In this respect, MAM may act as a timer to control the length of time that the Notch complex remains associated with the enhancer. By extension, MAM might contribute to the periodic expression of Notch target genes during somitogenesis through its potential effects on the disassembly of the Notch enhancer complex.

Our data indicate that CBF1 acts in concert with MAM to control the proteolytic turnover of the ICD in vivo (Fig. 6B). Importantly, both MAM and CBF1 appear to be stable upon coexpression with the ICD, and thus it appears that the ICD can be destabilized independently of its interacting partners. The requirement for CBF1 may reflect its ability to enhance binding of MAM to the ICD (Petcherski and Kimble 2000b), or alternatively CBF1 might be needed to target the Notch enhancer complex to DNA. We show that the stability of a mutant ICD protein lacking the PEST domain is unaffected by coexpression with MAM and CBF1 (Fig. 6A), and that turnover is accompanied by increased phosphorylation of the ICD (Fig. 7). Importantly, we find that the MAM TAD2 domain is necessary for both enhanced phosphorylation and turnover of the ICD. Because p300 has been shown to be critical for the regulated turnover of the p53 transactivator by MDM2 (Grossman et al. 1998; Zhu et al. 2001), it will be important to assess whether recruitment of p300 by MAM may similarly be required for proteolytic degradation of the ICD. Nevertheless, it is clear that recruitment of $\mathrm{CBP} / \mathrm{p} 300$ through the MAM TAD1 region is not sufficient to couple activation with turnover of the Notch ICD under the conditions examined here.

Thus the TAD2 region is required for MAM to promote the phosphorylation of its two associated factors, CBP/p300 and the Notch ICD. Because MAM does not possess intrinsic ICD protein kinase activity (data not shown), it is attractive to consider that the Notch ICD and $\mathrm{CBP} / \mathrm{p} 300$ may instead be targeted for phosphorylation by cyclin-dependent kinases that associate with the transcription complex (Price 2000; Orphanides and Reinberg 2002) and are recruited to the promoter by MAM (Fig. 8B). Phosphorylation events mediated by CDK7 and Srb10 (the CDK8 homolog in yeast) have been implicated in the proteolytic destruction of other enhancer factors 
(Vandel and Kouzarides 1999; Chi et al. 2001). The CDK9 subunit of the positive transcription elongation factor, P-TEFb, also associates with RNAPII (Price 2000), whereas CDK8 interacts with RNAPII as a component of human and yeast mediator complexes that have been variously implicated in activation and repression of transcription (Orphanides and Reinberg 2002). Another possibility is that the ICD is phosphorylated by a protein kinase that associates with MAM directly. It remains to be determined whether the MAM-induced phosphorylation is accompanied by increased ubiquitination of the ICD, and whether the degradation of the ICD we observe is caused by ubiquitin-dependent proteolysis such as that described for the nuclear Sel-10 ubiquitin ligase (Gupta-Rossi et al. 2001; Oberg et al. 2001; Wu et al. 2001). It will also be important to learn whether modification of the ICD regulates its transcriptional activity, as has been observed for other transcription factors (Hirst et al. 1999; Salghetti et al. 2001; Vincent et al. 2001; Ostendorff et al. 2002), and whether these steps may ultimately be coupled to disassembly of the Notch enhancer complex and turnover of the Notch ICD (Fig. 8C).

In summary, we show that MAM is an essential component of the Notch enhancer complex in vitro as well as in vivo. The human MAM protein recruits $\mathrm{p} 300 / \mathrm{CBP}$ to the Notch enhancer complex and controls the stability of the Notch ICD through the action of its unique Cterminal activation domain. Further studies will be needed to evaluate whether these properties are shared among the various MAM proteins in different species, and to learn how MAM-induced phosphorylation of the ICD and CBP/p300 proteins is coordinated with the regulation of Notch transcription.

\section{Materials and methods}

Plasmids and expression of recombinant proteins

Full-length human Mastermind (cDNA clone KIAA0200) and mutants were subcloned into pGEX-KG, a modified form of the PGEX-2T vector (Pharmacia) by standard PCR methods to yield constructs encoding in-frame fusions with glutathione- $S$-transferase (GST). Full-length MAM and 1-301MM mutants were subcloned into the CS2-MT vector by standard PCR methods to generate a Mastermind construct in frame with a C-terminal myc tag. GST-CBF1 was a generous gift of Lynne Vales (UMDNJ) and was expressed as described (Olave et al. 1998). The generation of the GST-ICD constructs was described previously (Wettstein et al. 1997). Protein expression was induced by addition of IPTG to a final concentration of $0.2 \mathrm{mM}$ and incubation at $37^{\circ} \mathrm{C}$ for an additional $4 \mathrm{~h}$. GST fusion proteins were purified as described (Tutter et al. 2001). Full-length human histidine-tagged p300 was expressed and purified from baculovirus-infected Sf9 cells as described previously (Kraus et al. 1999).

\section{DNA-binding experiments}

EMSA were carried out with the high-affinity CBF1 binding site from the HES1 promoter (CTAGGTTACTGTGGGAAAGAAA GTCC) in a final reaction volume of $15 \mu \mathrm{L}$ containing $20 \mathrm{mM}$ HEPES at pH 8.0, $50 \mathrm{mM} \mathrm{KCl}, 2.5 \mathrm{mM}$ EDTA, $8 \mathrm{mM} \mathrm{MgCl}_{2}, 5$
mM Spermidine, 30-35 $\mathrm{\mu g} / \mathrm{mL}$ poly(dIdC), $250 \mu \mathrm{g} / \mathrm{mL}$ BSA, $0.025 \%$ NP-40, and $15 \%$ glycerol.

\section{GST protein affinity-selection experiments}

First, $15 \mu \mathrm{L}$ of glutathione-sepharose 4B (Pharmacia) was equilibrated in GST-pulldown buffer $(50 \mathrm{mM}$ Tris at $\mathrm{pH} 7.9,120 \mathrm{mM}$ $\mathrm{KCl}, 0.5 \%$ NP-40, 2 mM DTT, $0.1 \mathrm{mM}$ PMSF, $2 \mathrm{mg} / \mathrm{mL}$ benzamidine, $1 \mu \mathrm{g} / \mathrm{mL}$ Pepstatin A, $4 \mu \mathrm{g} / \mathrm{mL}$ Leupeptin, $10 \mu \mathrm{g} / \mathrm{mL}$ Aprotinin, $20 \mu \mathrm{g} / \mathrm{mL}$ Soybean Trypsin Inhibitor) and combined with $100 \mu \mathrm{g}$ of purified recombinant GST alone or the indicated GST-tagged recombinant protein in a final volume of $300 \mu \mathrm{L}$ of GST-pulldown buffer $\left(4^{\circ} \mathrm{C}\right.$ for $\left.1 \mathrm{~h}\right)$. Excess unbound GST or GST-fusion protein was removed by briefly washing the beads with $300 \mu \mathrm{L}$ of GST-pulldown buffer. In a separate reaction, 150 $\mu \mathrm{L}$ of HeLa nuclear extract $(8-10 \mathrm{mg} / \mathrm{mL})$ was combined with $550 \mu \mathrm{L}$ of GST-pulldown buffer and precleared once over $15 \mu \mathrm{L}$ of glutathione sepharose $4 \mathrm{~B}\left(4^{\circ} \mathrm{C}\right.$ for $\left.1.5 \mathrm{~h}\right)$, and once over $20 \mu \mathrm{L}$ of the aforementioned GST-bound glutathione sepharose 4B $\left(4^{\circ} \mathrm{C}\right.$ for $\left.1.5 \mathrm{~h}\right)$. The precleared nuclear extract was then incubated with the aforementioned GST-fusion-bound glutathione sepharose at $4^{\circ} \mathrm{C}$ for $4 \mathrm{~h}$ with constant mixing. The depleted supernatant was discarded, and the beads were briefly washed 3 times with $300 \mu \mathrm{L}$ of HEGNO.3M (20 mM HEPES at $\mathrm{pH}$ 8.0, 0.2 mM EDTA, $10 \%$ gycerol, $0.3 \mathrm{M} \mathrm{KCl}, 0.1 \% \mathrm{NP}-40$ ) and once with $300 \mu \mathrm{L}$ of HEG0.1M (20 mM HEPES at $\mathrm{pH} 8.0,0.2 \mathrm{mM}$ EDTA, $10 \%$ glycerol, $0.1 \mathrm{M} \mathrm{KCl}$ ). The beads were then combined with $20 \mu \mathrm{L}$ of SDS-PAGE loading buffer, boiled, and electrophoresed through an $8 \%$ acrylamide SDS gel. Proteins were visualized by staining with silver. Association of p300 with GST-Mastermind constructs was detected by Western blotting with p300 (C20) antibody (Santa Cruz). Following the GST-pulldown reaction and Coomassie staining, proteins bands were excised, destained in acetonitrile and ammonium bicarbonate buffer, and subjected to in gel tryptic digest. A portion of the concentrated tryptic fragments was analyzed by MALDI-TOF with Reflectron, and proteins were identified by database comparison (Scripps Proteomics/Mass Spec Facility, La Jolla, CA).

\section{In vitro chromatin assembly and transcription}

Chromatin assembly was performed essentially as described (Bulger and Kadonaga 1994). pNRE contains eight copies of a consensus CBF1-binding site upstream of HIV-1 sequences (-79 to +80 , relative to the RNA start site) in a luciferase vector. For chromatin reconstitution of pNRE, $1.25 \mu \mathrm{g}$ of supercoiled plasmid DNA was used in each $250-\mu \mathrm{L}$ chromatin assembly reaction. Unless indicated, the GST-CBF1, GST-ICD, and GSTMAM were incubated with the pNRE template during chromatin assembly. Following assembly, $20-\mu \mathrm{L}$ aliquots of the chromatin reaction were incubated with HeLa cell nuclear extract and $25 \mathrm{ng}$ of nonchromatin $\alpha$-globin DNA at $30^{\circ} \mathrm{C}$ for 30 min. Transcription and DNase I footprint reactions on chromatin were carried out as described previously (Tutter et al. 2001).

\section{Chromatin HAT assay}

Chromatin was assembled in the presence of GST-CBF1, GSTICD, and GST-MAM as indicated. After assembly, $100 \mu \mathrm{L}$ of chromatin was purified on a Sepharose CL4B gel filtration column as described (Mizuguchi and Wu 1999). Then $20 \mu \mathrm{L}$ of purified chromatin was incubated $+/-$ p300 in the presence of 5 $\mu \mathrm{M}{ }^{14} \mathrm{C}$-acetyl CoA (Amersham) at $30^{\circ} \mathrm{C}$ for $1 \mathrm{~h}$. Reactions were stopped by boiling in SDS-PAGE loading buffer and analyzed on a $15 \%$ SDS-PAGE gel. The gel was fixed and treated with fluo- 
rography enhancing solution (Amersham) prior to drying and exposure to autoradiography

\section{Xenopus embryo RNA injection and in situ hybridization}

Embryos were obtained from Xenopus laevis adult frogs by hormone-induced egg-laying and in vitro fertilization using standard methods. Synthetic RNAs for injection into embryos were generated for full-length or deleted forms of human MAM by inserting the appropriate open reading frame from the cDNA clone KIAA0200 into the CS2 ${ }^{+} \mathrm{MT}$ vector. Templates for Notch$\mathrm{ICD}, \mathrm{XSu}(\mathrm{H}) \mathrm{DBM}$, and a nuclear-localized form of $\beta$-galactosidase (nlacZ) were described previously (Wettstein et al. 1997). Albino embryos at the two-cell stage were injected into one animal blastomere with $0.2-1 \mathrm{ng}$ of test RNAs, along with nlacZ RNA (500 pg) as a tracer. At early neurulae stages, injected embryos were fixed, stained for $\beta$-galactosidase activity with 5-bromo-4-chloro-3-indolyl- $\beta$-galactopyranoside (X-Gal), and then processed for whole-mount in situ hybridization for $\mathrm{N}$-tubulin expression as described previously using digoxigeninlabeled antisense riboprobes. (Lamar et al. 2001). At least 30 injected embryos were analyzed for each test RNA, in two independent experiments, and the phenotypes reported occurred in $>80 \%$ of the embryos. Embryos injected with just $n L a c Z$ served as a negative control.

\section{Indirect immunofluorescence and deconvolution microscopy}

All manipulations were done at room temperature. HeLa cells were grown on glass coverslips and transfected with $1 \mu \mathrm{g}$ of CS2-Mastermind DNA using Effectene (QIAGEN) according to the manufacturer's protocol. Cells were fixed for immunofluorescence $24-72 \mathrm{~h}$ after transfection with $3 \%$ paraformaldehyde, $0.05 \%$ glutaraldehyde in PBS for $20 \mathrm{~min}$, followed by $3 \mathrm{~min}$ of incubation with $0.1 \mathrm{M}$ glycine/PBS. Fixed cells were permeabilized with $0.2 \%$ Triton X-100 in PBS for 20 min and blocked in $2 \%$ BSA, $0.2 \%$ Triton X-100 for 20 min. The cells were incubated for $1 \mathrm{~h}$ with primary antibodies, followed by $40 \mathrm{~min}$ of incubation with appropriate secondary antibodies. The monoclonal antibodies 9E10 against the myc-epitope were purchased from Covance/Babco and used at a 1:2000 dilution. Rabbit antiCBP (A22) and anti-p300 (C20; Santa Cruz Biotechnology) were used at dilutions of 1:200 and 1:500, respectively. The primary antibodies were detected by secondary antibodies conjugated to Alexa 594 (goat anti-mouse IgG conjugate) or Alexa 488 (goat anti-rabbit IgG conjugate; Molecular Probes) at dilutions of 1:1000. Before mounting the cells onto slides using Vectashield mounting media (Vector Laboratories), the cell nucleus was counterstained with DAPI. Immunofluorescence images were collected as a Z-series with Olympus IX70 Microscope and deconvolved with Deltavision Software for image acquisition and processing (deconvolution). Adobe Photoshop (Adobe System) was used for image presentation, each image representing a single Z-section after deconvolution.

\section{Cell culture and Western analysis}

HeLa or C2C12 cells were grown in DMEM containing $10 \%$ FBS, L-glutamine, penicillin, and streptomycin. For transfection, 60,000 cells were plated in each well of 24-well dishes and transfected the next day in serum-free medium with $2 \mu \mathrm{g}$ of DNA per well using the PEI reagent (Fluka) according to published protocols (HeLa cells) or with 500 ng of DNA using lipofectamine (GIBCO BRL; C2C12 cells). In general, each effector constituted $\sim 20 \%$ of the total DNA transfected, which was normalized to a constant amount with carrier CS2 vector. Cells were washed in PBS and lysed in $400 \mu \mathrm{L}$ of ice-cold lysis buffer (25 mM Tris at $\mathrm{pH} 7.5,300 \mathrm{mM} \mathrm{NaCl}, 1 \%$ Triton X-100, $1 \mathrm{mM}$ PMSF, $1 \mu \mathrm{M}$ pepstatin, $1 \mu \mathrm{M}$ leupeptin, $1 \mathrm{mM}$ sodium vanadate, and $50 \mathrm{mM} \mathrm{NaF)} 48 \mathrm{~h}$ after transfection. Cell lysates were cleared by centrifugation at $4^{\circ} \mathrm{C}$ for $15 \mathrm{~min}$ at $15,000 \mathrm{rpm}$. Cell lysates were analyzed for the Myc-tagged proteins by Western blotting using the 9E10 myc mouse monoclonal antibody.

\section{Phosphatase treatment of $293 \mathrm{~T}$ and C2C12 extracts}

The 293T cells were transiently transfected with pCDNA empty vector, 1-301MM-myc, or 1016MM-myc, using calcium phosphate precipitation. Cell extracts were prepared $48 \mathrm{~h}$ after transfection and immunoprecipitated with anti-CBP antibody (Santa Cruz). Immunoprecipitated complexes were untreated or resuspended in phosphatase buffer and treated with $0.2 \mathrm{U}$ of acid phosphatase or $0.2 \mathrm{U}$ of acid phosphatase plus $1 \mathrm{mM}$ sodium vanadate. After these reactions were incubated at $37^{\circ} \mathrm{C}$ for $1 \mathrm{~h}$, cell lysis buffer was added, and the immune complexes were pelleted, resuspended in SDS-PAGE sample buffer, and loaded on a $6 \%$ gel. The immunoprecipitated complexes were detected by Western blotting with an anti-CBP antibody. C2C12 cells were transfected with myc-tagged forms of ICD, CBFI, and MAM as indicated, and cell extracts prepared $48 \mathrm{~h}$ after transfection were immunoprecipitated with an anti-myc antibody. The isolated proteins were treated with phosphatase as for 293T cells. The immunoprecipitated complexes were detected by Western blotting with an anti-myc antibody (9E10).

\section{Acknowledgments}

We thank Lynne Vales for the GST-CBF1 plasmid, and Lee Kraus and James Kadonaga for the p300 baculovirus expression vector. These studies were funded by grants from the NIH to K.A.J. and C.K. C.J.F. is supported by the Cancer Research fund of the Damon Runyan-Walter Winchell Foundation Fellowship (DRG-1610), and I.T. is funded by the Mac S. Rau Foundation.

The publication costs of this article were defrayed in part by payment of page charges. This article must therefore be hereby marked "advertisement" in accordance with 18 USC section 1734 solely to indicate this fact.

\section{References}

Ait-Si-Ali, S., Ramirez, S., Barre, F.X., Dkhissi, F., MagnaghiJaulin, L., Girault, J.A., Robin, P., Knibiehler, M., Pritchard, L.L., Ducommun, B., et al. 1998. Histone acetyltransferase activity of CBP is controlled by cycle-dependent kinases and oncoprotein E1A. Nature 396: 184-186.

Anderson, A., Robey, E., and Huang, Y. 2001. Notch signaling in lymphocyte development. Curr. Op. Genet. Dev. 11: 554560.

Artavanis-Tsakonas, S., Rand, M.D., and Lake, R.J. 1999. Notch signaling: Cell fate control and signal integration in development. Science 284: 770-776.

Bulger, M. and Kadonaga, J.T. 1994. Biochemical reconstitution of chromatin with physiological nucleosome spacing. Methods Mol. Genet. 5: 242-262.

Chi, Y., Huddleston, M., Zhang, X., Young, R., Annan, R., Carr, S., and Deshaies, R.J. 2001. Negative regulation of Gen 4 and Msn2 transcription factors by Srb10 cyclin-dependent kinase. Genes \& Dev. 15: 1078-1092.

Dou, S., Zeng, X., Cortes, P., Erdjument-Bromage, H., Tempst, P., Honjo, T., and Vales, L.D. 1994. The recombination signal sequence-binding protein RBP- $2 \mathrm{~N}$ functions as a tran- 
scriptional repressor. Mol. Cell. Biol. 14: 3310-3319.

Ferdous, A., Gonzalez, F., Sun, L., Kodadek, T., and Johnston, S. 2001. The 19S regulatory particle of the proteasome is required for efficient transcription elongation by RNA polymerase II. Mol. Cell 7: 981-991.

Greenwald, I. 1998. LIN-12/Notch signaling: Lessons from worms and flies. Genes \& Dev. 12: 1751-1762.

Grossman, S., Perez, M., Kung, A., Joseph, M., Mansur, C., Xiao, Z., Kumar, S., Howley, P., and Livingston, D. 1998. p300/ MDM2 complexes participate in MDM2-mediated p53 degradation. Mol. Cell 2: 405-415.

Gupta-Rossi, N., Le Bail, O., Gonen, H., Brou, C., Logeat, F., Six, E., Ciechanover, A., and Israel, A. 2001. Functional interaction between SEL-10, an F-box protein, and the nuclear form of activated Notch1 receptor. I. Biol. Chem. 276: 3437134378.

Helms, W., Lee, H., Ammerman, M., Parks, A.L., Muskavitch, M.A., and Yedvobnick, B. 1999. Engineered truncations in the Drosophila mastermind protein disrupt Notch pathway function. Dev. Biol. 215: 358-374.

Hirst, M., Kobor, M.S., Kuriakose, N., Greenblatt, J., and Sadowski, I. 1999. GAL4 is regulated by the RNA polymerase II holoenzyme-associated cyclin-dependent protein kinase SRB10/CDK8. Mol. Cell 3: 673-678.

Hsieh, J., Zhou, S., Chen, L., Young, D., and Hayward, S. 1999. CIR, a corepressor linking the DNA binding factor CBF1 to the histone deacetylase complex. Proc. Natl. Acad. Sci. 96: $23-28$.

Hubbard, E., Dong, Q., and Greenwald, I. 1996. Evidence for physical and functional association between EMB-5 and LIN-12 in Caenorhabditis elegans. Science 273: 112-115.

Hubbard, E., Wu, G., Kitajewski, J., and Greenwald, I. 1997. sel-10, a negative regulator of lin-12 activity in Caenorhabditis elegans, encodes a member of the CDC4 family of proteins. Genes \& Dev. 11: 3182-3193.

Ito, T., Ikehara, T., Nakagawa, T., Kraus, W., and Muramatsu, M. 2000. p300-mediated acetylation facilitates the transfer of histone H2A-H2B dimers from nucleosomes to a histone chaperone. Genes \& Dev. 14: 1899-1907.

Kamura, T., Sato, S., Haque, D., Liu, L., Kaelin, W.G., Jr., Conaway, R.C., and Conaway, J.W. 1998. The elongin BC complex interacts with the conserved SOCS-box motif present in members of the SOCS, ras, WD-40 repeat, and ankyrin repeat families. Genes \& Dev. 12: 3872-3881.

Kao, H.Y., Ordentlich, P., Koyano-Nakagawa, N., Tang, Z., Downes, M., Kintner, C.R., Evans, R.M., and Kadesch, T. 1998. A histone deacetylase corepressor complex regulates the Notch signal transduction pathway. Genes \& Dev. 12: 2269-2277.

Kitagawa, M., Oyama, T., Kawashima, T., Yedvobnick, B., Kumar, A., Matsuno, K., and Harigaya, K. 2001. A human protein with sequence similarity to Drosophila mastermind coordinates the nuclear form of notch and a CSL protein to build a transcriptional activator complex on target promoters. Mol. Cell. Biol. 21: 4337-4346.

Kraus, W., Manning, E., and Kadonaga, J. 1999. Biochemical analysis of distinct activation functions in p300 that enhance transcription initiation with chromatin templates. Mol. Cell. Biol. 19: 8123-8135.

Krebs, L.T., Deftos, M.L., Bevan, M.J., and Gridley, T. 2001. The Nrarp gene encodes an ankyrin-repeat protein that is transcriptionally regulated by the Notch signaling pathway. Dev. Biol. 238: 110-119.

Kurooka, H. and Honjo, T. 2000. Functional interaction between the mouse notch 1 intracellular region and histone acetyltransferases PCAF and GCN5. I. Biol. Chem.
275: $17211-17220$.

Lai, E.C. 2002. Protein degradation: Four e3s for the notch pathway. Curr. Biol. 12: R74-R78.

Lamar, E., Deblandre, G., Wettstein, D., Gawantka, V., Pollet, N., Niehrs, C., and Kintner, C. 2001. Nrarp is a novel intracellular component of the Notch signaling pathway. Genes \& Dev. 15: 1885-1899.

Lau, O.D., Kundu, T.K., Soccio, R.E., Ait-Si-Ali, S., Khalil, E.M., Vassilev, A., Wolffe, A.P., Nakatani, Y., Roeder, R.G., and Cole, P.A. 2000. HATs off: Selective synthetic inhibitors of the histone acetyltransferases p300 and PCAF. Mol. Cell 5: 589-595.

Mizuguchi, G. and Wu, C. 1999. Nucleosome remodeling factor NURF and in vitro transcription of chromatin. Methods Mol. Biol. 119: 333-342.

Morel, V., Lecourtois, M., Massiani, O., Maier, D., Preiss, A., and Schweisguth, F. 2001. Transcriptional repression by Suppressor of Hairless involves the binding of a HairlessdCtBP complex in Drosophila. Curr. Biol. 11: 789-792.

Mumm, J.S. and Kopan, R. 2000. Notch signaling: From the outside in. Dev. Biol. 228: 151-165.

Oberg, C., Li, J., Pauley, A., Wolf, E., Gurney, M., and Lendahl, U. 2001. The notch intracellular domain is ubiquitinated and negatively regulated by the mammalian sel-10 homolog. J. Biol. Chem. 276: 35847-35853.

Olave, I., Reinberg, D., and Vales, L.D. 1998. The mammalian transcriptional repressor RBP (CBF1) targets TFIID and TFIIA to prevent activated transcription. Genes \& Dev. 12: $1621-1637$.

Orphanides, G. and Reinberg, D. 2002. A unified theory of gene expression. Cell 108: 439-451.

Ostendorff, H.P., Pelrano, R.I., Peters, M.A., Schluter, A., Bossenz, M., Scheffner, M., and Bach, I. 2002. Ubiquitination-dependent cofactor exchange on LIM homeodomain transcription factors. Nature 416: 99-103.

Oswald, F., Tauber, B., Dobner, T., Bourteele, S., Kostezka, U., Adler, G., Liptay, S., and Schmid, R.M. 2001. p300 acts as a transcriptional coactivator for mammalian notch-1. Mol. Cell. Biol. 21: 7761-7774.

Petcherski, A.G. and Kimble, J. 2000a. LAG-3 is a putative transcriptional activator in the $\mathrm{C}$. elegans Notch pathway. $\mathrm{Na}$ ture 405: 364-368.

2000b. Mastermind is a putative activator for Notch. Curr. Biol. 10: R471-R473.

Pourquie, O. 1999. Notch around the clock. Curr. Op. Genet. Dev. 9: 559-565.

Price, D.H. 2000. P-TEFb, a cyclin-dependent kinase controlling elongation by RNA polymerase II. Mol. Cell. Biol. 20: 26292634.

Qiu, L., Joazeiro, C., Fang, N., Wang, H.Y., Elly, C., Altman, Y. Fang, D., Hunter, T., and Liu, Y.C. 2000. Recognition and ubiquitination of Notch by Itch, a hect-type E3 ubiquitin ligase. J. Biol. Chem. 275: 35734-35737.

Salghetti, S., Caudy, A., Chenoweth, J., and Tansey, W. 2001 Regulation of transcriptional activation domain function by ubiquitin. Science 293: 1651-1653.

Smoller, D., Friedel, C., Schmid, A., Bettler, D., Lam, L., and Yedvobnick, B. 1990. The Drosophila neurogenic locus mastermind encodes a nuclear protein unusually rich in amino acid homopolymers. Genes \& Dev. 4: 1688-1700.

Tansey, W. 2001. Transcription activation: Risky business. Genes \& Dev. 15: 1045-1050.

Tutter, A., Fryer, C., and Jones, K. 2001. Chromatin-specific regulation of LEF-1- $\beta$-catenin transcription activation and inhibition in vitro. Genes \& Dev. 15: 3342-3354.

Vandel, L. and Kouzarides, T. 1999. Residues phosphorylated by 
TFIIH are required for E2F-1 degradation during S-phase. EMBO J. 18: 4280-4291.

Vincent, O., Kuchin, S., Hong, S.P., Townley, R., Vyas, V.K., and Carlson, M. 2001. Interaction of the Srb10 kinase with Sip4, a transcriptional activator of gluconeogenic genes in Saccharomyces cerevisiae. Mol. Cell. Biol. 21: 5790-5796.

Wettstein, D.A., Turner, D.L., and Kintner, C. 1997. The Xenopus homolog of Drosophila Suppressor of Hairless mediates Notch signaling during primary neurogenesis. Development 124: 693-702.

Wu, G., Lyapina, S., Das, I., Li, J., Gurney, M., Pauley, A., Chui, I., Deshaies, R., and Kitajewski, J. 2001. SEL-10 is an inhibitor of notch signaling that targets notch for ubiquitin-mediated protein degradation. Mol. Cell. Biol. 21: 7403-7415.

Wu, L., Aster, J.C., Blacklow, S.C., Lake, R., Artavanis-Tsakonas, S., and Griffin, J.D. 2000. MAML1, a human homologue of Drosophila mastermind, is a transcriptional co-activator for Notch receptors. Nat. Genet. 26: 484-489.

$\mathrm{Xu}, \mathrm{T} .$, Rebay, I., Fleming, R.J., Scottgale, T.N., and ArtavanisTsakonas, S. 1990. The Notch locus and the genetic circuitry involved in early Drosophila neurogenesis. Genes \& Dev. 4: 464-475.

Yamamoto, N., Yamamoto, S., Inagaki, F., Kawaichi, M., Fukamizu, A., Kishi, N., Matsuno, K., Nakamura, K., Weinmaster, G., Okano, H., et al. 2001. Role of Deltex-1 as a transcriptional regulator downstream of the Notch receptor. $J$. Biol. Chem. 276: 45031-45040.

Zhou, S., Fujimuro, M., Hsieh, J.J., Chen, L., Miyamoto, A., Weinmaster, G., and Hayward, S. 2000. SKIP, a CBF1-associated protein, interacts with the ankyrin repeat domain of NotchIC to facilitate NotchIC function. Mol. Cell. Biol. 20: 2400-2410.

Zhu, Q., Yao, J., Wani, G., Wani, M., and Wani, A. 2001. Mdm2 mutant defective in binding p300 promotes ubiquitination but not degradation of p53. J. Biol. Chem. 276: 29695-29701. 


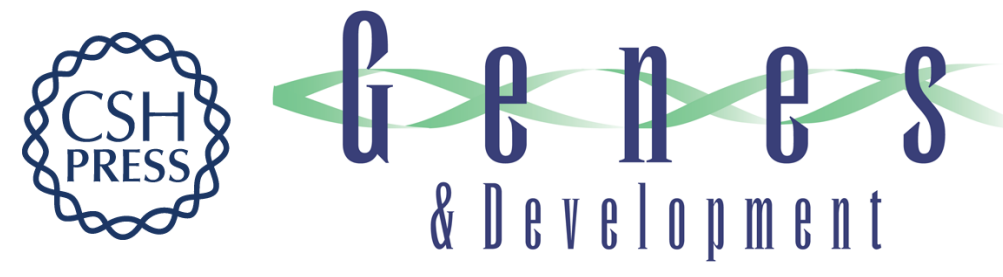

\section{Mastermind mediates chromatin-specific transcription and turnover of the Notch enhancer complex}

Christy J. Fryer, Elise Lamar, Ivana Turbachova, et al.

Genes Dev. 2002, 16:

Access the most recent version at doi:10.1101/gad.991602

References

This article cites 51 articles, 31 of which can be accessed free at: http://genesdev.cshlp.org/content/16/11/1397.full.html\#ref-list-1

License

Email Alerting

Receive free email alerts when new articles cite this article - sign up in the box at the top Service right corner of the article or click here.

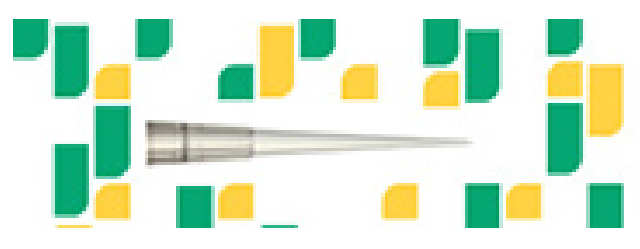

Focused on your science. 